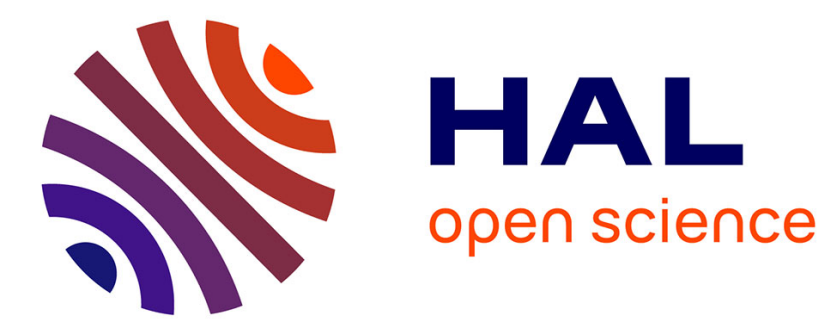

\title{
De l'aide à la responsabilisation
}

Jeanne Lazarus

\section{To cite this version:}

Jeanne Lazarus. De l'aide à la responsabilisation: L'espace social de l'éducation financière en France. Genèses. Sciences sociales et histoire, 2013, 4 (93), pp.76-97. 10.3917/gen.093.0076 . hal-01524049

\section{HAL Id: hal-01524049 \\ https://hal-sciencespo.archives-ouvertes.fr/hal-01524049}

Submitted on 17 May 2017

HAL is a multi-disciplinary open access archive for the deposit and dissemination of scientific research documents, whether they are published or not. The documents may come from teaching and research institutions in France or abroad, or from public or private research centers.
L'archive ouverte pluridisciplinaire HAL, est destinée au dépôt et à la diffusion de documents scientifiques de niveau recherche, publiés ou non, émanant des établissements d'enseignement et de recherche français ou étrangers, des laboratoires publics ou privés.

\section{(a)(1) $\$$}

Distributed under a Creative Commons Attribution - NonCommercial - NoDerivatives| 4.0 


\title{
De l'aide à la responsabilisation. \\ L'espace social de l'éducation financière en France
}

\author{
Jeanne Lazarus
}

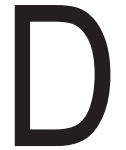

epuis une dizaine d'années, un nouveau terme a fait son entrée parmi les grands problèmes sociaux auxquels les gouvernements du monde doivent faire face : la financial literacy'. L'équivalent français seraitl' « alphabétisme financier », supposant l'existence d'un illettrisme financier, mais l'expression est le plus souvent traduite par «éducation financière », traduction un peu incomplète car le terme anglais désigne un niveau de compétence plutôt qu'une action éducative. Les pays précurseurs sont le Royaume-Uni, les États-Unis et la Nouvelle Zélande, pays de retraites par capitalisation et de prestations sociales limitées aux moins fortunés où la prise en charge des risques sociaux est largement du res-sort des ménages. Ces pays ont mis en place des politiques massives d'éducation financière: des agences publiques y pilotent des campagnes télévisées, l'introduc-tion de l'éducation financière dans les programmes scolaires, l'ouverture de points d'accueil budgétaires, etc. Sont visés en priorité les membres des classes moyennes salariées qui doivent planifier leur retraite, leurs assurances santé, le financement des études de leurs enfants ou un éventuel chômage, le tout à travers des produits boursiers structurellement soumis à de fortes incertitudes. Le besoin d'élever le niveau de compétence financière par des dispositifs pédagogiques de formation, d'apprentissage et d'éducation des populations des pays développés ${ }^{2}$ est mis en avant par l'OCDE depuis le début de la décennie 2000. Dans plusieurs rapports (OCDE 2005; 2011), le comité en charge des marchés financiers, des assurances et des retraites s'est inquiété du problème de financement des retraites par capi-talisation dans les pays ayant adopté ce système. L'organisation joue depuis, par le biais de conférences internationales, nombreux rapports, guides de bonnes pra-tiques, enquêtes de grande ampleur, un rôle de lanceur d'alerte auprès des gou-vernements sur la nécessité d'éduquer les populations non pas tant à certaines 
pratiques qu'à connaître les risques qu'elles auront à affronter. Ces efforts ont été couronnés de succès puisqu'en décembre 2011, le G20 a approuvé les grands prin-cipes pour l'éducation financière que l'OCDE avait établis. En outre, la fameuse enquête PISA de l'OCDE, qui se présente comme un étalon des compétences scolaires des jeunes de 15 ans des pays développés, intègre depuis 2012 un module de culture financière.

Dans ce cadre, la France est un cas intéressant parce qu'elle semble ne pas avoir pris le train de la financial literacy. L'OCDE a publié en 2013 un bilan d'étape (OCDE 2013) dans lequel les rédacteurs se félicitent du fait que 45 pays aient déjà lancé des politiques de financial literacy. Cependant, la France fait partie des cinq derniers pays membres de l'OCDE « en train de réfléchir » au lancement d'une politique d'éducation financière. Le ministère de l'Économie a annoncé en octobre 2013 qu'il lançait une stratégie nationale et un rapport a été commandé.

Un discours multiforme sur le « besoin d'éducation du consommateur de pro-duits financiers » se développe dans l'Hexagone depuis quelques années. Il est porté par des hommes politiques, des hauts fonctionnaires, des représentants du secteur bancaire comme de celui du travail social. Ce besoin d'éducation est essentiellement pensé en direction des populations pauvres ou en difficulté financière, quand la pro-motion de la financial literacy de l'OCDE est surtout destinée aux classes moyennes salariées. Trois raisons expliquent cette spécificité française. La première est institu-tionnelle : l'Étatprovidence français, s'il est en cours de rétractation, reste l'un des plus protecteurs du monde et les retraites fonctionnent par répartition. Le problème de la financial literacy pensé ailleurs en termes de maîtrise des risques liés au dévelop-pement de produits d'épargne et d'assurances individuelles sophistiqués s'exprime en France par le thème de la " frilosité » des épargnants réticents aux placements boursiers. Mais ce thème reste peu développé et n'a pas fait l'objet d'une politique publique. En effet, la seconde raison est que les difficultés dans les rapports des ins-titutions financières avec les particuliers ont été problématisées en France autour du surendettement (Salomon 1995 ; Plot 2011). Enfin, plus récemment, la promotion de l'éducation financière a été concomitante à la mise en place des microcrédits per-sonnels, à l'origine d'un rapprochement paradoxal entre le monde bancaire et celui de l'accompagnement social. Depuis des positions très différentes, ces acteurs pen-sent l'éducation à l'argent comme une solution aux problèmes existants : mauvaises relations entre clients et conseillers bancaires, ventes de produits inutiles, problèmes budgétaires des clients, endettement excessif, etc.

II s'est ainsi créé ce que Lilian Mathieu (2007 : 133) nomme un espace social : « un univers de pratique et de sens relativement autonome à l'intérieur du monde social » dont les acteurs sont unis par des relations d'interdépendance. L'espace français de l'éducation financière se caractérise par des interconnaissances, des partenariats entre acteurs bancaires, associatifs et publics, mais aussi par une struc-turation des positions de chacun et des déplacements nécessités par un travail commun entre monde bancaire et monde de l'accompagnement social. 
L'objet de cet article est de comprendre l'origine de ce rapprochement entre des acteurs qui ne manquent pas de se critiquer et de s'affronter dans d'autres arènes. Jacques Lagroye, Bastien François et Frédéric Sawicki (2002 : 518) écri-vaient : « Problématiser une question, ce n'est pas seulement dire ce qu'elle est, c'est indissociablement dire qui est appelé à intervenir, de quelle manière et avec quels moyens. " II nous semble donc nécessaire d'analyser la façon dont les questions budgétaires et financières touchant aux particuliers en France sont mises en pro-blème pour comprendre la place donnée actuellement à l'éducation financière.

La première problématisation est celle de la Fédération des banques françaises (FBF), l'organisme professionnel des banques. Pour elle, l'ensemble des tensions dans les agences bancaires et les problèmes bancaires des clients sont la conséquence de leur inculture financière. Cette problématisation par l'inculture place entièrement la responsabilité sur les clients, pour lesquels l'unique solution est d'être mieux éduqués financièrement.

La contre-problématisation, miroir de celle-ci, est celle de l'inclusion bancaire telle que la défend Georges Gloukoviezoff, socio-économiste auteur de L'exclusion bancaire (2010), dont les écrits sont beaucoup lus et discutés par les membres de l'espace social de l'éducation budgétaire, qui l'invitent souvent et pour lesquels il rédige des enquêtes sur l'impact des dispositifs d'accompagnement des clients ou de microocrédit (Gloukoviezoff et Lazarus 2007 ; Gloukoviezoff et Palier 2009 ; Gloukoviezoff et Rebière 2013). À ses yeux, promouvoir l'éducation financière est une manœuvre destinée à masquer la responsabilité des dispositifs et produits bancaires dans les difficultés des clients. II est au diapason des travaux sociolo-giques britanniques néo-foucaldiens qui dénoncent la promotion de la financial literacy comme un moyen supplémentaire de définition des individus comme des entrepreneurs d'eux-mêmes afin de leur faire accepter de supporter individuelle-ment des risques sociaux autrefois pris en charge collectivement (voir par exemple Langley et Leaver 2012).

La troisième problématisation peut être qualifiée de pragmatique : l'observa-tion des pratiques budgétaires des individus montre leur manque de savoir-faire pratiques, et leur transmettre les bonnes façons de faire pourra leur éviter de nom-breux problèmes. L'essentiel est donc la qualité des formations dispensées et de ses effets. Cette problématisation est beaucoup moins homogène qu'elle n'en a l'air. Elle est à la fois celle de l'OCDE et des experts qui participent à la rédaction de ses rapports et de ses recommandations politiques, pour lesquels l'éducation financière peut être considérée comme une politique économique à part entière, et celle d'acteurs impliqués dans l'éducation financière, qui estiment qu'elle n'est utile qu'en coordination avec d'autres formes d'intervention sociale et ne doit pas se substituer à la régulation. Pour eux, elle n'est pas un instrument politique mais un savoir-faire indispensable à la vie quotidienne contemporaine.

Comment ces problématisations différentes se rencontrent-elles et sont-elles mises en acte par les acteurs de l'éducation budgétaire? Les polarisations de cet 


\section{L'enquête}

Cet article se base sur une enquête de terrain de plusieurs années auprès de l'OCDE et des acteurs français de l'éducation financière (banques, services sociaux, associations). Pour une partie, l'enquête a consisté dans l'observation de séances collectives ou individuelles de formation à l'argent et d'entretiens avec les formateurs comme avec les responsables des entités concernées. S'y ajoute l'observation de nombreuses conférences, colloques et autres forums organisés par les acteurs impliqués dans l'éducation budgétaire en France et à l'étranger pour les conférences internationales de l'OCDE sur le sujet.

Le positionnement social des acteurs interviewés ne sera ici restitué que par rapport à leur institution. En effet, l'article vise à présenter les pôles existants et les positionnements d'entités au sein d'un espace social en voie de construction; ce sont donc les déplacements nécessités par cette construction qui seront au cœur de l'article. En outre, étant donné la forte interconnaissance entre les différents acteurs cités, la préservation de l'ano- nymat implique de ne pas exposer trop d'informations personnelles.

Une autre partie de l'enquête relève de l'observation participante. J'ai rédigé deux rapports pour deux entités impliquées dans ce champ. Le premier traitait de l'impact des microcrédits mis en place par le Secours catholique (Goukoviezoff et Lazarus 2007), ce travail relativement ancien m'a permis de voir les débuts de la mise en place $\mathrm{du}$ « partenariat » entre banques et monde associatif 3 , et surtout de nouer des contacts avec des personnes devenues ensuite importantes dans le développement de l'éducation financière. L'autre rapport était destiné à mesurer la satisfaction des « partenaires », c'est-à-dire des structures faisant appel à une association importante dans le champ de l'éducation financière, nommée " Finances \& Pédagogie » (Lazarus 2013). Ce second rapport, fondé sur une enquête par questionnaire (31) et des entretiens (23) a été l'occasion de comprendre com-ment la question de l'éducation budgétaire et bancaire était posée dans le monde du travail social.

espace social s'expliquent également par les types de ressources que peuvent mobi-liser les acteurs : ressources économiques pour les uns, légitimité d'intervention envers les publics en difficulté pour les autres, accès aux pouvoirs publics enfin. Les dynamiques de circulation et d'utilisation de ces ressources sont au cœur des relations entre les membres de cet espace.

La première partie de l'article montrera la façon dont travailleurs sociaux, associations caritatives et banques ont collaboré depuis une dizaine d'années et le rôle fédérateur du thème de l'éducation budgétaire dans ce cadre.

Dans un second temps, à travers l'analyse des partenariats mis en place par Finances \& Pédagogie, association d'éducation financière importante que nous présenterons alors, nous observerons les types de déplacement dans les définitions du travail et de l'accompagnement social qu'accompagne et implique la diffusion de pratiques d'éducation financière.

\section{L'espace social de l'éducation financière et budgétaire}

En France, un acteur est officiellement en charge de l'éducation financière : I'Institut pour l'éducation financière du public (IEFP), dépendant de l'Autorité des marchés financiers (AMF), dont la mission est d'améliorer le niveau général de compétences financières de la population. II est lié aux instances économiques les plus légitimes: AMF, Banque de France, ministère de l'Économie et des 
Finances, ministère de l'Éducation nationale. Pourtant, l'IEFP, connu principale-ment par son site internet « la finance pour tous », est marginal dans l'espace social de l'éducation financière, car il travaille avant tout avec l'Éducation nationale et n'est que peu présent auprès des services sociaux et associations caritatives qui sont les acteurs centraux de cet espace social, comme nous allons le montrer. La péda-gogie de l'argent est prise en charge par les pouvoirs publics dans les pays qui pro-meuvent la financial literacy à travers des agences dédiées (en Nouvelle-Zélande), les autorités de surveillance des marchés financiers (en Grande-Bretagne) ou des agences de protection des consommateurs (aux États-Unis). Ce n'est pas le cas en France. L'espace de l'éducation financière y est polarisé entre le « marchand » et le « social » (1), ce qui s'explique par la genèse de celui-ci dans le monde du microcré-dit (2), selon une problématisation pragmatique éloignée d'une problématisation par l'inculture économique et financière.

\section{"C'est comme la réunification de l'Allemagne de l'Est et de l'Allemagne de l'Ouest "}

Cetelem organise depuis quelques années des groupes de travail avec ce qu'il nomme des « parties prenantes » : représentants d'associations de consommateurs, de syndicats, d'associations caritatives et représentants d'organismes publics; L'Agence nouvelle des solidarités actives (ANSA) ${ }^{4}$ a piloté entre 2011 et 2013 une expérimentation destinée à évaluer les effets de l'éducation à l'épargne, en réunis-sant plus d'une dizaine d'acteurs, banques, associations caritatives, spécialistes de l'éducation budgétaire et autres centres communaux d'action sociale ; le cabinet de conseil Athling management, qui a rédigé des rapports pour le ministère de l'Éco-nomie et a beaucoup influencé la mise en place de la loi Lagarde ${ }^{5}$, invite à ses petits déjeuners annuels les représentants des banques, pouvoirs publics et associations caritatives ; le Crédit municipal organise chaque année depuis 2009 des « forums d'échange entre les acteurs sociaux et bancaires » sur des thèmes liés au microcré-dit ou aux finances des personnes en difficulté, etc. Le public de tous ces espaces est très similaire, les participants se reconnaissent, se saluent et poursuivent des conversations parfois engagées lors du forum ou de la journée précédente. Dans les couloirs certains lancent des phrases comme : « Je ne suis pas étonné qu'Untel se soit positionné ainsi, il était dans son rôle. »

Cette interconnaissance, le positionnement attendu des uns et des autres en fonction de leurs institutions, la multiplicité des rencontres et les partenariats liant ces acteurs permettent de considérer qu'il existe un espace social les réunissant autour de l'accompagnement budgétaire et de l'éducation financière.

Cet espace s'organise autour de deux axes structurants : le capital économique et le degré d'insertion des entités dans le monde marchand, d'une part ; de l'autre, leur niveau perçu de désintéressement, matière de leur capital symbolique (Bourdieu 2000). La possession de ces deux types de capitaux est relativement antinomique et c'est ce qui explique dans bien des cas la collaboration entre les 
deux groupes d'acteurs. Comme le disait la responsable d'une association impor-tante : "Voir les banques organiser des conférences où elles invitent les travailleurs sociaux et les associations du coin, c'est comme la réunification de l'Allemagne de l'Est et de l'Allemagne de l'Ouest. »

Les banques possèdent le capital économique, à n'en pas douter. Elles financent, à travers leurs politiques de responsabilité sociale, des programmes d'éducation financière. Pour autant, elles ne souhaitent pas être réduite au rôle de portemonnaie. Elles estiment au contraire posséder des compétences essentielles pour s'impliquer dans l'éducation financière : "Si l'on veut apprendre à faire du pain, on va chez un boulanger, si l'on veut apprendre l'argent, on va vers les institu-tions financières ", dit ainsi la responsable d'une fondation liée à une banque. Les banques s'estiment entièrement légitimes pour faire de l'éducation financière car elles connaissent les produits bancaires qu'elles vendent et ont une vue imprenable sur les budgets de leurs clients. Pourtant, malgré leur sentiment de compétence et de légitimité technique, il leur manque la légitimité sociale. Pour le dire autrement, si la problématisation unique de l'éducation financière était une problématisation pragmatique et fondée sur l'inculture des clients, elles pourraient maîtriser seules ce domaine. Toutefois, pour l'heure, ce n'est pas le cas.

La Société générale a lancé un site internet appelé ABC Banque en novembre 2011 pour les enfants de 6 à 11 ans. Ce site développé par une société spécialisée dans les publications pour enfants n'avait pas de « partenariat» avec des acteurs du monde social ou caritatif. Dans « l'espace parents » du site, les concepteurs ont pris la précaution d'écrire : «ABCBanque.fr est un site pédagogique qui ne contient ni offre commerciale ni publicité. Notre ambition est de développer un site référent en matière d'éducation à la finance $»^{6}$. Cette phrase n'a pas empêché les attaques contre le site lors de son lancement, par des articles de journaux, des commentaires d'internautes l'accusant de vouloir enrégimenter les enfants et les faire adhérer à la Société générale.

Inversement, la Fédération des banques françaises (FBF), l'organisme professionnel des banques, qui a une activité d'éducation budgétaire importante (site inter-net ayant reçu 2 millions de visites en 2012, publication de mini-guides et partena-riats avec des Centres communaux d'action sociale), indique sur un certain nombre de ses publications que des conseillères en économie sociale et familiales (CESF) ou des associations ont participé à leur rédaction ${ }^{7}$ et n'hésite jamais à évoquer ses partenaires associatifs et sociaux. Ainsi, la FBF tient-elle compte de la problémati-sation fondée sur l'exclusion financière, qui implique que la cible de ces programmes soient les personnes les moins fortunées, d'où découle que les acteurs légitimes pour intervenir dans ce champ relèvent du monde de l'accompagnement social.

Les associations et les services sociaux possèdent peu de capital économique mais détiennent une très forte légitimité d'intervention auprès des publics en dif-ficulté, qui pourrait être qualifiée de capital symbolique. La notion de "capital symbolique ", que nous reprenons ici en nous référant à Esquisse d'une théorie de 
la pratique de Pierre Bourdieu (2000), où il désigne le travail de masquage du caractère intéressé des pratiques et des échanges pour les faire apparaître comme relevant du don et de la réciprocité, ne peut être opérant qu'à la condition d'en avoir précisé l'origine. Dans cet espace, c'est le désintéressement perçu des acteurs qui en est la matière première. Je parlerai donc de capital de désintéressement : désintéressement financier, puisque les accompagnateurs sociaux ne nouent pas de relations marchandes avec ceux qu'ils accompagnent, mais aussi désintéresse-ment comme authenticité de l'engagement. Ce capital est constitué des signaux marquant l'éloignement des enjeux commerciaux, comme le bénévolat, les actions à but non lucratif ou, pour les associations de consommateurs, l'autonomie finan-cière complète par rapport aux annonceurs. Enfin, l'objectif poursuivi par ces enti-tés se rapporte à un bien commun relevant de la cité civique ou de la cité domes-tique - contrairement aux entreprises qui poursuivent également un bien commun associé, lui, à la cité marchande ou industrielle (Boltanski et Thévenot 1991).

Le désintéressement se caractérise par l'impossible imputation d'une visée utilitariste ; il a donc une composante réputationnelle importante. Aucune entité n'est à l'abri d'un procès en intéressement. Les associations se voient parfois repro-cher leur intéressement politique : telle association chercherait à " se placer ", à " prendre la main ». Ces concurrences restent légitimes tant qu'elles sont associées à des différences de points de vue ou de valeurs. Elles deviennent illégitimes si elles sont décrites comme des concurrences de notoriété, voire des concurrences d'ordre marchand (telle association se placerait sur ce thème pour obtenir des subventions ou des contrats avec des institutions financières). Le désintéressement des pou-voirs publics n'est pas non plus à l'abri des soupçons : telle politique ne viserait-elle pas en réalité à faire des économies ? À faire accepter des transformations sociales néfastes pour les plus pauvres ? À privilégier telle catégorie de la population, voire telle corporation?

Mais ces procès en intéressement sont, dans l'espace de l'éducation financière, principalement adressés aux institutions bancaires. Même si certaines banques tra-vaillent à euphémiser leurs pratiques marchandes (Moulévrier 2002), étant donné la centralité des institutions financières dans l'établissement du capitalisme, leurs pratiques sont structurellement l'objet d'une lecture relevant de ce que Bourdieu appelle l'« économisme » : leur réduction à un « système régi par les lois du calcul intéressé, de la concurrence ou de l'exploitation » (2000 : 350). Dès lors, pour tenter d'acquérir un capital de désintéressement, les banques créent souvent des entités distinctes, à but non lucratif (comme la Fondation Cetelem ou bien Finances \& Pédagogie pour les Caisses d'épargne, sur lesquelles nous reviendrons) ; elles mettent en place des chartes déontologiques - par exemple les conseillers de Finances \& Pédagogie ne parlent jamais de la Caisse d'épargne et ont supprimé les logos de la banque de tous leurs supports pédagogiques. D'autres stratégies consistent à trouver des entités extérieures pour certifier les efforts de responsabi-lité sociale d'entreprise. C'est ce qu'a fait Cetelem avec l'entreprise Vigeo, agence 
de notation sociale, qui a attesté du caractère « responsable » de son crédit renouve-lable - cela participe au «travail de crédibilisation que Cetelem entreprend depuis 2004 autour du "crédit responsable $₫$ " $"$ ", ainsi que le proclame un communiqué de presse de 2012. Le marquage de ces frontières est délicat : elles doivent être suffisamment étanches pour garantir l'intégrité des actions caritatives, mais suffisamment poreuses pour que l'entreprise mère bénéficie du gain d'image que ces « bonnes actions » peuvent fournir.

Toutefois, le fait qu'elles soient des entreprises commerciales ou y soient liées empêche que ces entités puissent acquérir une quantité élevée de capital de désintéressement : lorsqu'elles investissent des terrains d'accompagnement social, le " dévoilement » de leur " véritable » moteur qu'est la « volonté de faire du profit » est une possibilité permanente. Par exemple, lors d'un des forums de discussion entre les différents partenaires, une jeune femme, salariée du Secours catholique, s'est levée. D'une voix nouée par l'indignation, elle s'est insurgée contre l'intrusion de Cetelem dans leurs activités d'accompagnement social : « On n'a pas les mêmes objectifs, eux et nous. Nous, on veut vraiment aider les gens, eux ils veulent faire duprofit. »

Les associations caritatives qui coopèrent avec des banques risquent de perdre en désintéressement : ne sont-elles pas en train de se " vendre » aux banques ? Le marquage des frontières entre le " social » et le commercial est une nécessité dans cet espace. Les acteurs qui y sont le plus légitimes sont dès lors ceux qui, malgré les déplacements nécessaires à leur collaboration avec des banques, ont conservé leur capital de désintéressement ou en possèdent suffisamment pour fréquenter presque sans danger des institutions financières. C'est le cas du Secours catho-lique, qui bénéficie d'une telle légitimité en tant qu'association caritative qu'il peut nouer des partenariats avec des banques et même avec des établissements spé-cialisés de crédit comme Cofinoga ou Cetelem sans risquer de perdre son statut. Toutefois, même ces entités doivent se prémunir contre d'éventuelles critiques, internes - nous l'avons vu plus haut - ou externes.

C'est pourquoi le monde de l'accompagnement social est très attaché aux marques de son autonomie vis-à-vis de ses partenaires bancaires, qui est une façon de reconstituer son capital symbolique tout en rappelant la hiérarchie du désinté-ressement. Ainsi, lorsque la Croix-Rouge a organisé, le 22 octobre 2012, une jour-née intitulée « Argent, Banque, Budget » destinée à fédérer les acteurs du Manifeste pour l'inclusion bancaire en France des populations fragiles, signé un an plus tôt par elle-même, le Secours catholique et l'UNCCAS', les banques n'étaient pas invitées - même si ces trois entités ont des partenariats avec nombre d'entre elles.

Ces entités sont dominantes dans l'espace du désintéressement et possèdent donc le pouvoir symbolique leur permettant de « constituer le donné en l'énon-çant, d'agir sur le monde en agissant sur la représentation du monde » (Bourdieu et Wacquant 1992 : 123). Elles disposent du pouvoir de désigner et de sélection-ner, parmi les entités dominées (c'est-à-dire les banques), celles dont l'engagement 
est authentique et les autres, et donc de distribuer du capital symbolique. Ainsi, dans l'interaction citée plus haut, lorsqu'une jeune femme travaillant pour le Secours catholique a mis en cause la probité de Cetelem, c'est un représentant de la fondation Abbé Pierre qui lui a répondu : s'il ne faut pas être naif, a-t-il affirmé, et si chacun sait qui il est et reste dans son rôle (c'est-à-dire si les banques ne font pas semblant d'être des associations caritatives et que les associations ne se mettent pas à raisonner de façon économiciste), alors un travail en commun efficace peut avoir lieu.

Cet échange permet de comprendre que seules les entités au fort capital de désintéressement sont en mesure de certifier celui de celles qui en ont moins (la représen-tante de Cetelem présente à ce forum a également pris la parole mais celle-ci était beaucoup moins efficace dans cet espace social que celle du représentant de la fonda-tion Abbé Pierre), et également que la quantité de capital de désintéressement que les entreprises financières peuvent acquérir est structurellement faible. Et c'estici que l'on peut mesurer l'intérêt de la question de la problématisation des compétences finan-cières pour les banques : dans l'espace social tel qu'il est actuellement organisé, elles ne pourront que rester dominées. En revanche, si des déplacements s'opèrent et que la définition des grandeurs (Boltanski et Thévenot 1991) se transforme, notamment si la compétence technique (la problématisation pragmatique et fondée sur l'inculture) acquiert une légitimité supérieure à celle de la compétence caritative (la problémati-sation fondée sur l'exclusion financière), elles pourront alors s'élever.

Notons que si le plus souvent, une relation de proportionnalité inverse lie la possession du capital économique et celle du capital de désintéressement dans l'espace de l'éducation budgétaire, certains acteurs se détachent. C'est le cas du Crédit municipal de Paris et de la Banque postale : ces deux institutions finan-cières, l'une établissement public, l'autre récemment privatisée, possèdent un capi-tal de désintéressement bien supérieur aux autres banques (Moulévrier 2012). La banque postale conserve une image de banque « sociale » et le Crédit municipal de Paris est très lié aux services sociaux de la Ville de Paris, avec qui il a créé les Points solutions surendettement, services publics d'aide aux surendettés. Ainsi, alors que les organisateurs de la journée du manifeste pour l'inclusion bancaire y ont souli-gné leur choix de ne pas inviter les banques, les représentants du Crédit munici-pal étaient présents, signe qu'aux yeux des organisateurs caritatifs cette entité ne peut être qualifiée de "banque ». Ce positionnement ne manque pas de faire grin-cer des dents les organismes à l'image plus discutée : « le Crédit municipal, c'est une banque quand même. Ils font toujours comme s'ils étaient une association » (représentante RSE, Établissement spécialisé de crédit).

\section{L'effet déclencheur du microcrédit personnel}

Le rapprochement entre banques et monde de l'accompagnement social n'a toutefois rien d'évident : pourquoi ces acteurs aux systèmes de valeur et aux objec-tifs très différents se côtoient ou s'associent-ils ? Ce rapprochement trouve sa genèse dans la mise en place du microcrédit personnel. 
En 2005, le ministère de l'Économie a créé le Fonds de cohésion sociale, d'un montant de 130 millions d'euros, géré par la Caisse des dépôts, destiné notamment à garantir et promouvoir les microcrédits personnels : de 500 à 3000 euros pour un TEG moyen de $5 \%$ en 2012 (Gloukoviezoff et Rebière 2013), ces microcrédits sont accordés par des banques à des personnes en situation précaire mais accompagnées par des associations caritatives ou des services sociaux et doivent servir à des « projets » personnels ${ }^{10}$. Ils se distinguent ainsi des microcrédits professionnels destinés à finan-cer des entreprises. De nombreuses associations ont participé et participent à cette expérience. Le Secours catholique a été l'une des premières et des plus impliquées, mais les autres grandes associations caritatives y participent, ainsi que les centres communaux d'action sociale (CCAS) et des associations de taille plus modeste.

Ces organismes d'accompagnement social ont alors regardé bien plus attentivement qu'ils ne le faisaient jusque-là les budgets des personnes puisque les demandeurs devaient exposer en détail leur situation financière. Ces études budgétaires ont été souvent l'occasion de jugements négatifs sur la gestion des demandeurs : aux yeux des travailleurs sociaux et bénévoles, ces mauvais gestionnaires avaient besoin d'être éduqués pour mieux hiérarchiser leurs dépenses et apprendre à résister à toutes leurs envies. Par ailleurs, le dispositif de microcrédit impliquait que des bénévoles associatifs ou des travailleurs sociaux fassent de « l'accompa-gnement budgétaire ॥. II leur a alors été demandé d'acquérir des formes d'exper-tise concernant les produits bancaires et un savoir-faire pour « parler argent » avec les personnes accompagnées, en particulier pour quitter la position parfois trop moralisante et paternaliste qu'un certain nombre d'entre eux endossaient.

De multiples initiatives d'éducation budgétaire ont donc eu lieu à destination des bénéficiaires de microcrédit et des accompagnateurs. Certaines étaient organi-sées à l'intérieur même des associations par des bénévoles se chargeant de conce-voir des modules, d'autres en partenariat avec des organismes jugés compétents, le plus souvent liés à des banques. Cela ne s'est pas fait sans heurts : en 2006, lorsque le Secours catholique a été l'une des premières organisations caritatives à signer des partenariats avec les banques pour financer des microcrédits, de nombreux bénévoles s'indignèrent. Ce fut notamment compliqué en région Aquitaine car le partenaire local était Cofinoga (Gloukoviezoff et Lazarus 2007). Lorsque les banques partenaires étaient des banques de type mutualiste (Crédit mutuel, Crédit lyonnais ou Caisses d'épargne), les bénévoles avaient tendance à être plus conci-liants qu'avec des organismes perçus comme les prêteurs les moins scrupuleux et les plus dangereux pour les pauvres (Lazarus 2012).

Pour le monde associatif et du travail social, la mise en place du microcrédit n'a pas seulement été l'occasion de nouer ces partenariats parfois jugés scandaleux, ce fut aussi un moyen de rencontrer de nouvelles populations :

"Le CCAS s'est lancé dans un nouveau dispositif avec le microcrédit, et finalement c'est grâce à ce dispositif qu'on a pu faire le constat de certaines formes de difficultés 
économiques des ménages. Puisque le CCAS a bien entendu une connaissance de son territoire par l'analyse de ses besoins sociaux [...]. Sauf que le microcrédit nous a fait permis de toucher des nouveaux publics » (Conseillère en économie sociale et familiale, CCAS).

C'est également ce que dit cette responsable d'une association nationale d'aide alimentaire : «On a des personnes qui ne rentrent pas dans le barème [pour bénéfi-cier de l'aide alimentaire] mais qui viennent quand même chez nous parce qu'elles ont entendu parler de nous par le microcrédit, ou on essaie de les accompagner par un autre moyen. »

Ces personnes que les services sociaux ou les associations « ne rencontraient pas jusque-là » ont été classées par eux comme appartenant aux « classes moyennes fragiles ${ }^{11} »$ : catégorie définie comme regroupant des personnes non habituées à contacter les services sociaux mais recevant des salaires insuffisants pour faire face à leurs charges. Cette population est l'objet d'une grande attention des différents acteurs de l'éducation financière. Elle est importante pour ces acteurs sociaux d'abord parce qu'elle est politiquement légitime : elle cadre parfaitement avec la problématisation habituelle de l'intervention sur les questions financières par le prisme de la procédure de surendettement (Plot 2011) puisque ses membres sont des surendettés en puissance (faibles revenus mais charges élevées) et elle est décrite comme méritante. «Trop riches pour recevoir des aides et trop pauvres pour faire face ", ses membres ne font pas appel à la solidarité nationale, ne sont pas chroniquement assistés. Ils auraient honte de demander ; la preuve en est le fort taux de non-recours au RSA activité. Cette figure est l'image même de la "crise » économique, puisqu'« avant » elle pouvait s'en sortir mais ne le peut plus ${ }^{12}$.

La « population fragile » a en outre l'avantage de représenter des situations sur lesquelles il est possible d'agir par la pédagogie de l'argent. Lorsque les personnes ne touchent que le RSA : «On aura beau leur donner tous les conseils qu'on veut, c'est trop juste » dit une assistante sociale travaillant pour une association carita-tive. Inversement, des personnes recevant de petits salaires mais ayant des difficul-tés financières sont une « matière » sur laquelle agir.

L'apparition des populations fragiles dans l'espace d'intervention potentielle des services sociaux a fait naître le thème de leur " détection » : si certains se sont manifestés à travers les demandes de microcrédits, sans doute d'autres, dans des situations similaires, ne sont-ils pas repérés alors qu'ils sont en situation de danger financier. Les banques ont trouvé ici un terrain de légitimité, faisant valoir leurs compétences : elles ont accès aux comptes des personnes et ont des savoir-faire statistiques anciens et efficaces (Lazarus 2010). Par exemple, Cetelem a mis au point un algorithme pour repérer ses clients risquant d'avoir des impayés de crédit avant que des incidents n'aient lieu, qu'il a mis à la disposition de ses concurrents - à ce jour, plusieurs d'entre eux ont visité ses locaux et certains utilisent même l'algorithme de l'établissement de crédit pour détecter leurs propres clients fragiles. 
On le perçoit ici, le voisinage d'institutions bancaires et d'associations au fort capital de désintéressement ne se résume pas à un jeu à somme nulle où les banques gagneraient ce que les associations perdraient. Une telle représentation validerait une vision en « mondes hostiles » (Zelizer 2005) et ne tiendrait pas compte du processus central à l'œuvre, qui est un déplacement de la définition des besoins des personnes rencontrant des difficultés sociales. Les promoteurs de l'assurance vie au xix ${ }^{\mathrm{e}}$ siècle ont fait accepter leurs polices en transformant la définition de la « bonne mort » (Zelizer 1978). La transformation n'est pas aussi radicale ici. Pour autant, pour faire accepter leur participation au monde de l'éducation finan-cière, les institutions financières contribuent à la redéfinition du « bon » accom-pagnement social, des « bonnes » compétences que doivent acquérir les personnes accompagnées comme celles qui les accompagnent. Ces efforts produisent de nouvelles frontières et de nouvelles définitions de l'accompagnement social, qui redéfinissent les grandeurs respectives de chacun.

L'observation plus approfondie des partenariats noués par l'un des acteurs importants du champ nous permettra de comprendre les effets de la promotion de l'éducation budgétaire sur la conception de l'accompagnement social.

\section{Formation à l'argent et nouvelle description du travail social}

Finances \& Pédagogie est une association fondée en 1957 par les Caisses d'épargne afin de faire de l'éducation budgétaire dans une logique traditionnelle d'économie domestique. Voilà comment sa dirigeante - en place depuis une quin-zaine d'années après une carrière dans le monde bancaire - présente les racines de l'association:

«On a une existence de terrain : le livret de la ménagère, le mari qui dépense son argent en buvant sa bière... La femme qui compte le reste de l'argent pour la can-tine... Voilà, ça, c'était notre histoire à nous. ॥

Elle insiste : "Nous, on vient de l'épargne populaire », pour se distinguer de ceux qui viennent de " l'épargne financière », c'est-à-dire l'Institut d'éducation financière du public et la FBF. Cette distinction est d'autant plus nécessaire que Finances \& Pédagogie est liée à une banque - certes de tradition mutualiste et ayant des missions d'intérêt général, mais dont les pratiques d'agence n'ont rien à envier à ses collègues commerciales.

F\&P intervient aussi bien dans le cadre de la formation professionnelle qu'au-près de publics en difficulté, qui peuvent être aussi différents que des salariés de chantiers d'insertion, des jeunes travailleurs en foyer, des prisonniers, des résidents de centres d'hébergement, etc. Elle se situe donc au cœur de l'espace social de l'accompagnement budgétaire et de ses partenariats hybrides. Ses formateurs sont pour la plupart d'anciens salariés de la Caisse d'épargne, expérience d'où ils tirent 
leur expertise. Ils se définissent comme ayant un " profil plus social » que leurs anciens collègues - c'est-à-dire qu'ils aiment passer du temps à parler avec les clients, à réparer des découverts plutôt qu'à faire de la vente à tout crin. La diri-geante de Finances \& Pédagogie, comme les formateurs, considère qu'ils ont une compétence rare et utile : ils savent autant dialoguer avec le monde de la banque qu'avec celui de l'accompagnement social.

Le contenu des formations de Finances \& Pédagogie peut se résumer en deux grands axes : d'une part, un apport de connaissances (comment fonctionne un compte, un découvert, un crédit ou un plan d'épargne de retraite, selon l'intérêt de l'audience) ; d'autre part, des conseils de comportement : ouvrir les courriers de la banque, planifier, hiérarchiser, anticiper. Un des outils utilisés en formation qui, d'après les formateurs, a beaucoup d'effet sur les participants est la pyramide de Maslow, qui hiérarchise les besoins en cinq catégories, depuis les plus fon-damentaux - les besoins physiologiques puis de sécurité - vers les besoins les plus « sociaux » : besoin d'appartenance, d'estime et de s'accomplir, au sommet de la pyramide. Les « stagiaires " sont invités à classer leurs dépenses dans chacune de ces catégories, et à se rappeler qu'il faut d'abord assurer sa subsistance et sa sécurité avant d'acquérir des biens davantage tournés vers le statut social. Cette recommandation de hiérarchisation est extrêmement classique dans les conseils budgétaires. Par exemple, l'agence néo-zélandaise de promotion de la financial literacy se nomme Sorted, qui signifie trié, classé, hiérarchisé.

La position intermédiaire de Finances \& Pédagogie entre la banque et l'accompagnement social en fait un bon point d'entrée pour observer la place que l'éducation budgétaire prend ou pourrait prendre dans le travail social. J'ai été sollicitée par la responsable de l'association pour achever une enquête commencée par une salariée ayant dû quitter son poste, et dont l'objectif était de comprendre ce que les structures faisant appel à F\&P en attendaient. Pour la directrice, l'objectif était de montrer que l'éducation financière est désormais l'un des outils obligatoires de tout processus d'insertion du fait de la financiarisation de la vie quotidienne.

L'enquête s'est déroulée en deux temps : analyse des questionnaires (construits par la salariée de F\&P) puis entretiens auprès de 23 responsables des organismes faisant appel à l'association en Île-de-France destinés à comprendre le rôle que ces derniers veulent faire jouer à l'éducation budgétaire et financière ainsi que les tensions que cette notion est en mesure de résoudre pour eux. Ces organismes qui le plus souvent suivent des personnes en difficulté sociale sont de fait confrontés à leurs problèmes d'argent, soit directement - par exemple, retards de paiement de personnes logées en centre d'hébergement -, soit indirectement - par exemple dans des entreprises d'insertion ${ }^{13}$ qui effectuent un " suivi social global ", c'est-à-dire s'occupent d'aider les individus à obtenir leurs droits sociaux - accès aux soins médicaux, éventuellement au logement, etc. - et qui voient apparaître les problèmes budgétaires lors de demandes d'avance sur salaire. 
Or la plupart des travailleurs sociaux ne sont que peu voire pas formés à l'accompagnement budgétaire. Ils travaillent sur des questions qui touchent de très près au budget des personnes, auxquelles ils sont en général amenés à demander leurs ressources et leurs dépenses ; pour autant, leur action consiste principalement à ouvrir des droits ou à évaluer l'éligibilité des usagers aux aides existantes plutôt qu'à cher-cher à transformer les pratiques des individus. Dès lors, si comme dans d'autres domaines d'intervention, l'action des travailleurs sociaux peut être qualifiée de nor-mative (Serre 2009), cette normativité passe davantage par des jugements portés et l'effet concret de ces jugements sur l'accord ou le refus de mettre en place certaines aides, que par des techniques visant à transformer les pratiques budgétaires ${ }^{14}$.

Dans ce cadre, les responsables des structures font appel à F\&P aussi bien pour intervenir auprès des personnes accompagnées qu'auprès des travailleurs sociaux pour lutter contre ce que certains cadres de l'action sociale nomment leur « culture de l'assistanat » : trouver des aides plutôt qu'apprendre aux personnes à gérer leur budget. Cette insistance sur les projets des individus associée à l'individualisation des situations correspond aux transformations du travail social décrites par Nico-las Duvoux (2009). Les travailleurs sociaux, quelle que soit leur position indivi-duelle sur le sujet, se doivent de se conformer à ces injonctions, qui se traduisent par le durcissement des critères d'aide, des exigences beaucoup plus strictes de participation financière lors des hébergements et la description du cheminement des individus comme devant faire l'objet d'un projet.

Cette tension entre l'aide - souvent au cœur de l'engagement professionnel des travailleurs sociaux et de la définition de leur métier - et la responsabilisation des personnes accompagnées, si elle est constante dans le travail social, est d'autant plus visible lorsqu'il s'agit des questions budgétaires. Ainsi, le responsable d'un centre d'hébergement m'indique la différence entre l'ancienne « culture de l'aide » - «malheureusement» encore présente chez certains de ses subalternes - et la « nouvelle » culture de la responsabilisation : « Ils sont d'accord pour aider, mais pas pour responsabiliser, autonomiser avec l'argent. » Lorsqu'une personne se présente avec des difficultés de paiement de sa participation, dans l'« ancienne culture » le travailleur social recherchait des aides sociales pour combler son retard ; dans la « nouvelle », le travailleur social dresse un état des lieux budgétaires avec la per-sonne, établit un moratoire de remboursement avec elle afin qu'elle «prenne ses responsabilités ». La " modernité » de l'action sociale passe par la responsabilisa-tion, et en particulier la responsabilisation budgétaire des personnes. Pourtant, dans la réalité des suivis, les deux types d'intervention coexistent et mettent les travailleurs sociaux en situation de tension : s'ils adhèrent souvent au discours de responsabilisation, contre la figure de l'assisté (Duvoux 2009), leur honneur professionnel réside dans le soutien aux personnes. Les formations à l'argent de Finances \& Pédagogie les aident à combiner les deux.

Finances \& Pédagogie se positionne clairement du côté de la promotion de l'autonomie et de la responsabilisation. On peut lire sur sa plaquette de présenta- 
tion que F\&P encourage « la prise de responsabilité et l'autonomie financière ». Ce positionnement vient en soutien à ceux qui portent ce discours au sein des orga-nismes partenaires, le plus souvent en position d'encadrement, mais il est égale-ment en mesure d'apaiser pragmatiquement les tensions car l'association propose des outils aux personnes accompagnées pour s'adapter à cette exigence d'auto-nomisation. Ainsi, dans le questionnaire préalable à la campagne d'entretiens, 29 répondants sur 31 avaient indiqué que « l'action de F\&P est déterminante pour une meilleure autonomie dans la gestion du budget ».

Les entretiens ont permis de comprendre ce que les répondants entendaient par le terme " autonomie ». Pour certains, depuis une position moralisatrice, l'auto-nomie est l'opposé de l'assistanat. Son acquisition permet de « se prendre en main » et de « prendre conscience » de ses faiblesses et erreurs pour devenir un citoyen res-ponsable, comme l'évoque l'extrait d'entretien suivant avec une conseillère en éco-nomique sociale et familiale (29 ans, six ans d'ancienneté comme CESF) chargée d'accompagner des locataires HLM au bord de l'expulsion pour de lourds retards de loyer. Elle expose d'abord les raisons de la situation:

" C'est-à-dire que la personne ne s'organise pas de manière à transmettre les documents en temps et en heure, et se retrouve impactée par des frais. [...] La mauvaise gestion du budget, c'est lié aussi au fait que la personne n'a pas pris les dispositions pour organiser son budget. C'est-à-dire qu'il y a des personnes qui ont 40 ans, et elles n'ont toujours pas mensualisé leurs impôts sur le revenu ou leur taxe d'habitation. »

En outre, ces personnes « n'ont pas conscience de leur mauvaise gestion budgé-taire et renvoient la faute soit sur des institutions, soit sur la société ». L'atelier de Finances \& Pédagogie a, à ses yeux, transformé leurs " comportements » concrets, mais a surtout eu des effets sur l'attitude anciennement passive des locataires " en » impayés : " L'atelier a contribué à permettre à des personnes d'avoir une prise de conscience et de pouvoir prendre certaines dispositions au niveau de leur budget pour être en capacité de gérer. D'où le fait qu'elles ont évité l'expulsion. »

Mais l'autonomie, c'est aussi la possibilité d'augmenter les possibilités d'action des personnes (Eyraud 2013). C'est ce que souligne cette responsable d'un ESAT (établissement et service d'aide par le travail), centre d'aide aux personnes handicapées s'occupant spécifiquement de jeunes adultes entre 16 et 25 ans porteurs de lourds handicaps. Certains n'ont jamais acheté quoi que ce soit seuls, encore moins fait un chèque ou utilisé une carte bancaire. Lorsqu'ils sont dans l'ESAT, ils en sont salariés et reçoivent pour la première fois des revenus. Le maître mot de la structure est l'autonomisation, et les questions monétaires en font partie :

« Souvent c'est une occasion pour les parents de prendre conscience que leur fille ou leur fils va arriver à un moment charnière de leur vie où il va y avoir la prise d'auto-nomie pour le budget. Et du coup, il faut qu'ils lâchent un petit peu, qu'ils laissent le stagiaire faire des opérations sur lesquelles [la formatrice] les entraîne. Ils réagissent. Par exemple ils doivent payer leur titre de repas. Ils ont des carnets avec des tickets à 
payer, donc on les invite à ce moment-là, s'ils ont un chéquier, à apprendre à faire le chèque, à le faire eux-mêmes, à le faire vérifier par la famille s'il le faut, mais que ce soit eux qui fassent la démarche, dans le souci de la prise de l'autonomie. [...] souvent les parents pensent qu'ils ne sont pas capables. Là le jeune peut montrer à ses parents : je suis capable de faire un chèque. J'ai besoin encore de mes parents mais je suis en mesure de pouvoir le faire»

(Responsable ESAT).

L'autonomie est ici conçue comme une pratique, la capacité à faire soi-même un certain nombre des tâches de la vie quotidienne. Toutefois, enjoindre à l'auto-nomie c'est aussi décrire un rapport au monde et ce qu'est une « bonne vie ». Paul Ricoeur (2001) décrit ces exigences nouvelles sur les personnes encadrées par le travail social comme une demande de cohérence narrative. Dans le cas de l'argent, l'exigence d'autonomie est une exigence de cohérence dans le temps incarnée par des capacités de planification. II s'agit tout autant d'être en mesure de construire des projets que d'être capable de les intégrer dans une vie passée et future. Les CESF disent souvent qu'il faut « remettre à plat » les situations, décrivant des per-sonnes en difficulté qui n'ouvrent plus leurs relevés de compte, n'arrivant plus à compter et à " poser » leur budget. De même, les travailleurs sociaux ou bénévoles associatifs accompagnant les personnes surendettées déplorent souvent que les gens « ne [sachent] pas pourquoi ils se sont endettés ». Dans les deux cas, c'est un manque de cohérence narrative qui est pointé.

Dans l'expérience européenne SIMS (voir encadré) mesurant les effets de l'éducation budgétaire, les participants indiquent avant tout qu'ils ont appris de

\section{Apprendre l'épargne}

Le projet européen SIMS (Social Innovation on MicroSavings), avait pour objectif de mesurer l'efficacité de la pédagogie de l'argent en France, en Belgique et en Hongrie. Côté français, il était piloté par l'Agence nouvelle des solidarités actives fondée par Martin Hirsch, dont l'objec-tif est de « promouvoir des expérimentations sociales » et qui utilise en particulier les méthodes de l'évaluation ran-domisées (voir l'article d'Arthur Jatteau dans le présent numéro). Le dispositif français réunissait la plupart des acteurs de l'éducation financière: banques, associations d'accompagnement des personnes en difficulté budgé-taire, spécialistes de l'éducation et travailleurs sociaux. La cible était des apprentis en CFA (496 ont participé à l'étude) : un groupe recevait une formation à l'argent délivrée par des formateurs de Finances \& Pédagogie et centrée sur l'épargne, l'autre non. Leurs compétences et comportements étaient périodiquement mesurés. Pour la restitution finale, un film de témoignages a été réalisé.
On y écoute notamment Mohamed, 19 ans, apprenti, ayant reçu une formation. II y a appris « la valeur de l'argent », et « compris » qu'il est important d'épargner pour faire des projets. Avant il n'épargnait pas, dit-il, tout son budget passait dans les loisirs. Mais il sait désormais que « l'argent ça part vite et si on n'épargne pas on aura du mal à avancer dans la vie ». Grâce à l'épargne, affirme-il, il a pu passer son permis et a de nouveaux projets : acheter une meilleure voiture, plus tard un appartement. Au début du mois il prépare son budget, sépare ce qu'il peut dépenser en fonction des postes. Aujourd'hui, il est un " consommateur conscient ». Les témoins filmés dans les trois pays évaluent les bénéfices de l'expérience avec un mélange d'utilitarisme (l'épargne permet de faire face aux coups durs ou de partir en vacances) et de morale : en devenant de meilleurs planificateurs, ils sont devenus plus raisonnables, plus conscients, plus réfléchis. 
cette expérience la planification. Apprendre «la valeur de l'argent», c'est apprendre à l'inscrire dans le temps.

L'acquisition de l'autonomie est un moyen de retrouver de la cohérence nar-rative, et donc une forme de participation sociale : cette responsable d'un chantier d'insertion qui emploie des travailleurs du bâtiment, dont certains sont d'anciens SDF, donne une importance primordiale à la possibilité de faire des projets dans la vie des personnes qu'elle accompagne :

«Quand ils arrivent, ils sont dans le rien. C'est-à-dire qu'ils ne se projettent plus du tout. Ils n'ont pas de projets du tout, c'est de la survie au jour le jour. [...] Ce que j'ai-mais bien avec [le formateur] c'est qu'il leur disait : voilà, vous pensez qu'avoir des pro-jets c'est pas possible, donc en général ils disent tous : oui c'est pas possible. [Eh bien ?] si, on peut quand même d'une certaine façon » (Responsable entreprise d'insertion).

Pour elle, si l'éducation budgétaire contribue à lutter contre l'exclusion, ce n'est pas par des outils pratiques qui aideraient à sortir de la pauvreté par une meilleure gestion. C'est qu'en leur proposant des modules de formation à la banque et à l'argent, elle indique aux personnes accompagnées qu'elles sont aussi légitimes que les autres à être affiliées à cette institution sociale qu'est la banque. II est important pour elle de leur montrer que des « banquiers ${ }^{15}$ » en costume sont d'accord pour prendre du temps pour eux.

Finances \& Pédagogie offre donc un déplacement par rapport à l'accompagne-ment budgétaire traditionnel (qui consistait en la réforme de son budget et de soi-même) : l'association propose moins de redresser les comportements budgétaires que d'aider les personnes à participer au système bancaire, obligatoire dans une société financiarisée, et en ce sens de lutter contre l'exclusion bancaire. D'ailleurs, lorsque je demande aux formateurs ce qu'ils jugent être une formation réussie, ce n'est pas du contenu précis de ce qui est transmis ou retenu qu'ils parlent, mais de l'« énergie » qu'ils transmettent. L'un d'entre eux affirme qu'il veut faire « poser des actes » aux personnes qui suivent ses ateliers.

On entend ici le vocabulaire de l'« empowerment » (Bacqué et Biewener 2013) et la volonté de donner de la dignité aux individus en les décrivant comme des citoyens autonomes et capables d'agir sur leur destin. Dans un cadre où les accom-pagnateurs sociaux ont acté la diminution des aides sociales et des filets de sécu-rité collectifs, l'éducation budgétaire apparaît comme une solution et un horizon libérateur face à des situations douloureuses. Elle est également parfois considérée comme le moyen de lutter contre la domination bancaire en empêchant les indivi-dus de tomber dans les pièges que le marketing bancaire et les techniques de vente pourraient leur tendre. II peut ainsi y avoir une hybridation entre la problématisa-tion pragmatique et la problématisation fondée sur l'exclusion bancaire. L'éduca-tion financière pourrait être un levier permettant de mieux armer la critique contre les banques - et à un niveau individuel, pour des personnes pauvres, un levier leur donnant les savoir-faire et l'« autorisation » de discuter ce qui leur est proposé dans 
les agences bancaires. Elle répond donc à des enjeux que les travailleurs sociaux observent en accompagnant les personnes en difficulté et vient apaiser les tensions issues des deux types de normes professionnelles actuellement présentes dans le travail social : aide contre responsabilisation. Éduquer à la responsabilité est une façon de répondre à cette double exigence.

Toutefois, la promotion de l'éducation financière peut également avoir un effet de démobilisation d'une critique politique plus large, puisqu'elle est qualifiée d'utile lorsque les transformations politiques du travail social sont décrites comme inévitables, Deus ex machina sur lequel aucune prise n'est possible.

La place croissante de l'éducation financière au sein du travail social indique que le « budget » est en train de devenir une matière à part entière de l'accompa-gnement social et de la description de la vie des usagers. Une sorte de désencas-trement est à l'œuvre, qui consiste à supprimer le contexte social, voire le contexte financier dans lequel les personnes accompagnées gèrent leur budget, pour ne plus parler que de hiérarchisation, de choix et donc de responsabilité.

La question posée au début de l'article était de comprendre la spécificité de l'approche française de l'éducation financière qui passe par un rapprochement entre le monde de l'accompagnement social et le monde bancaire. Si la genèse de ce rapprochement ne se trouve pas dans la promotion de l'éducation finan-cière ellemême mais dans la conception des microcrédits personnels, l'éducation à l'argent fait figure de thème fédérateur entre des entités aux positionnements différents. Leur rapprochement masque les variations de problématisations : pour les uns, la promotion de l'éducation financière est un moyen politique permettant de mettre en lumière les " mauvais " comportements des consommateurs plutôt que ceux des acteurs bancaires, quand pour les autres, l'éducation financière est vue comme une aide pragmatique à apporter à des personnes qui sont forcées de participer à une société financiarisée sans avoir toujours les savoirs et savoir-faire leur permettant d'affronter les institutions financières et les entreprises de biens et de services avec lesquelles elles sont en contact (Perrin-Heredia 2009).

Les expériences internationales mais également françaises montrent que plus les publics visés sont " élevés » socialement et plus la problématisation fondée sur l'inculture est efficace. Ce cadrage se concentre sur les choix des consommateurs, pour leur retraite, leurs assurances santé, leur épargne et tout ce qui touche à la pré-voyance individuelle. Ces choix seraient de mauvaise qualité du fait d'informations insuffisantes, difficiles à trouver ou à comparer. La solution sera alors d'aider les consommateurs à améliorer le cadre dans lequel ils opèrent ces choix, par exemple en utilisant des techniques de nudge - terme anglais qui signifie « pousser du coude » et qui relève du « paternalisme libertarien » : il s'agit de faire agir les individus d'une façon précise en jouant sur leur " architecture de choix », par exemple en repensant 
les plans de retraite d'entreprise de sorte que les choix par défaut soient ceux dont les montants épargnés soient les plus élevés (Thaler et Sustein 2008 ; Duflo et Saez 2003). L'éducation financière est alors définie comme une affaire d'experts en écono-mie comportementale, voire de marketing social (Lusardi, Keller et Keller 2009) et de banquiers. En revanche, plus les publics sont financièrement en difficulté, moins les banques sont légitimes, mais paradoxalement, plus elles peuvent gagner en capi-tal de désintéressement. Si les pédagogies économiques ayant le vent en poupe se présentent toutes comme porteuses d'empowerment (terme parfois traduit par « pou-voir d'agir »), elles sont différemment pensées selon les publics : la distance entre les concepteurs des campagnes et les cibles est plus grande pour les classes moyennes salariées et plus faibles pour les plus pauvres, puisque pour ces derniers, l'éducation budgétaire et financière est presque toujours pensée en lien avec de l'accompagne-ment et de la proximité. En revanche, pour les classes moyennes, elle est davan-tage pensée comme une politique économique et comme relevant de formes de gouvernements des conduites économiques, qui peuvent donc avoir lieu à distance (Dubuisson-Quellier 2014).

La jeune femme du Secours catholique citée en première partie s'insur-geait contre le partenariat noué avec Cetelem en sous-entendant que ces actions n'étaient qu'une façon de capter les publics pauvres pour leur vendre du crédit. Pourtant, si l'entreprise cherche un gain économique dans son intervention auprès des pauvres, celuilà n'est pas direct : il ne s'agit pas de faire prendre des crédits aux personnes qui auront bénéficié de programmes d'éducation financière financés par la fondation Cetelem - bien au contraire, cela discréditerait entièrement le projet. Le bénéfice pour les banques de leur participation à l'espace de l'éducation finan-cière est bien différent. Si elles tentent évidemment d'améliorer ainsi leur image, leur visée est plus large : ces actions leur confèrent de la légitimité pour participer au cadrage politique du surendettement et des difficultés financières des « popula-tions fragiles » et pour contribuer à en définir les modes et les lieux de résolution, à l'extérieur des agences bancaires, dans des salles de formation à l'argent. 


\section{Ouvrages cités}

Bacqué, Marie-Hélène et Carole Biewener.2013. L'empowerment, une pratique émancipatrice. Paris, La Découverte.

Boltanski, Luc et Laurent Thévenot. 1991. De la justification. Paris, Gallimard.

Bourdieu, Pierre. 2000. Esquisse d'une théorie de la pratique. Paris, Seuil.

Bourdieu, Pierre et Loïc Wacquant. 1992. Réponses. Pour une anthropologie reflexive. Paris, Seuil.

Dubuisson-Quellier, Sophie (éd.). 2014. Gouverner les conduites. Paris, Presses de SciencesPo (à paraître).

Duflo, Esther et Emmanuel Saez. 2003. «The role of information and social interactions in retirement plan decisions: evidence from a randomized experiment », The Quarterly Journal of Economics, vol. $118, n^{\circ} 3: 815-842$.

Duvoux, Nicolas. 2009. L'autonomie des assistés. Paris, Puf.

Eyraud, Benoît. 2013. Protéger et rendre capable. Toulouse, Eres.

Gloukoviezoff, Georges. 2010. L'Exclusion bancaire. Paris, Puf.

Gloukoviezoff, Georges et Jeanne Lazarus. 2007. «Évaluation d'impact des Crédits projet personnel du Secours catholique. Rapport intermédiaire pour le Secours catholique», rapport final, LEFI- Université Lyon 2.

Gloukoviezoff, Georges et Jane Palier. 2009. « Évaluation de l'efficacité organisationnelle du dispositif de microcrédits personnels du Crédit municipal de Paris», rapport final, Lyon, 2GRecherche.

Gloukoviezoff, Georges et Nicolas Rebière. 2013. «Étude d'impacts du microcrédit personnel garanti», 2G Recherche, COMPTRASEC, Caisse des dépôts et consignation.

\section{Lagroye, Jacques, Bastien François} et Frédéric Sawicki (éd.). 2002. Sociologie politique. Paris, Presses de Sciences Po et Dalloz.
Langley, Paul et Adam Leaver. 2012. «Remaking retirement investors» Journal of Cultural Economy, vol.5, n $4: 473-488$.

Lazarus, Jeanne. 2010. «La bancarisation du crédit». Entreprises et Histoire, $n^{\circ}$ 59: 28-40.

-2012. L'épreuve de l'argent. Banques, banquiers, clients. Paris, Calmann-Levy.

-2013. «La perception des interventions de Finances \& Pédagogie par ses partenaires». Paris, Finances \& Pédagogie.

Lusardi, Annamaria, Punam Keller et Adam Keller. 2009. «New ways to make people save: a social marketing approach », in Annamaria Lusardi (éd.), Overcoming the Saving Slump: How to Increase the Effectiveness of Financial Education and Saving Programs. Chicago, University of Chicago Press.

Mathieu, Lilian. 2007. «L'espace des mouvements sociaux». Politix, nº77: 131-151.

Moulévrier, Pascale. 2002. Le mutualisme bancaire. Le Crédit mutuel de l'Église au marché.Rennes, Pur.

- 2012. « Les structures sociales du marché bancaire en France », Revue française de socio-économie, vol. $1, n^{\circ} 9: 23-41$.

OCDE. 2005. «Improving financial literacy: analysis of issues and policies», OECD Publishing.

OCDE et Russia's G20 Presidency. 2013. " Advancing national strategies for financial education », OECD Publishing.

OCDE. 2011. «Improving financial education efficiency: OECD-Bank of Italy Symposium on financial literacy», OECD Publishing.

- 2013. «Cadre d'évaluation de la culture financière du cycle PISA 2012», in Cadre d'évaluation et d'analyse du cycle PISA 2012. Compétences en mathématiques, en compréhension de l'écrit, en sciences, en résolution de problèmes et en matières financières, Éditions OCDE.

Perrin-Heredia, Ana. 2009. «Les logiques sociales de l'endettement: gestion des comptes domestiques en milieux populaires $»$. Sociétés contemporaines, $n^{\circ} 76$ : 95-116. 
-2010. « Logiques économiques et comptes domestiques en milieux populaires", thèse de sociologie, Université de Reims ChampagneArdenne.

Plot, Sébastien. 2011. «Les enjeux d'une mise en risque. La construction

du surendettement comme problème public

(1989-2010)», thèse de science politique, Université

Paris-Dauphine.

Ricœur, Paul. 2001. «Autonomie et vulnérabilité » in Paul Ricœur, Le Juste, t. 2. Paris, Esprit : 85-106.

Salomon, Danielle. 1995. «La transformation du système bancaire français. L'exemple

du segment du crédit à la consommation », thèse de sociologie, IEP Paris.

Serre, Delphine. 2009. Les coulisses de l'État social. Enquête sur les signalements d'enfant en danger. Paris, Raisons d'Agir.
Schwartz, Olivier. 2009. «Vivons-nous encore dans une société de classes? Trois remarques sur la société française contemporaine», La Vie des idées.

En ligne: http://www.laviedesidees.fr/Vivonsnous-encore-dans-une.html (consulté le 8/01/2014)

Thaler, Richard et Cass Sustein. 2008. Nudge Improving Decisions About Health, Wealth, and Happiness. Yale, Yale University Press.

Zelizer, Viviana. 1978. «Human Values and the Market: The Case of Life Insurance and Death in 19thCentury America », American Journal of Sociology, vol.84, $n^{\circ} 3: 591-610$.

-2005. The Purchase of Intimacy. Princeton, Princeton University Press.

\section{NOTES}

1. Je remercie les relecteurs Vincent Gayon, Benjamin Lemoine, Etienne Nouguez et Etienne Ollion pour leurs précieux commentaires qui ont grandement aidé à améliorer ce texte, dont je reste seule responsable des éventuelles erreurs ou omissions.

2. Et par extension des pays en voie de développement, notamment à travers ce que l'OCDE nomme des " conférences de dissémination » sur le sujet de la financial literacy, qui ont régulièrement lieu dans des pays non membres.

3. Les membres du comité d'évaluation de cette expérience de microccrédit, présidé par JeanMichel Belorgey - conseiller d'État, notamment à l'origine de la convention "Belorgey ", qui a permis que les assurances acceptent de prendre en charge des clients atteints de maladie chronique - étaient des membres du Secours catholique, des représentants d'entités relevant de l'économie sociale et solidaire comme l'ADIE ou le Crédit coopératif, des hauts fonctionnaires impliqués dans des missions de lutte contre la pauvreté, des représentants de la Caisse des dépôts et d'anciens banquiers.

4. L'ANSA, organisme fondé par Martin Hirsch, dont l'objectif est de «promouvoir des expérimen- tations sociales " et qui utilise en particulier les méthodes de l'évaluation randomisée (voir l'article d'Arthur Jatteau sur le sujet).

5. La loi sur le crédit à la consommation, dite «loi Lagarde ", est entrée en vigueur à partir de 2010 , avec notamment des mesures destinées à limiter les excès du crédit renouvelable.

6. http://www.abcbanque.fr/qui-sommes-nous, consulté le 19 décembre 2013.

7. Par exemple, pour le «guide pratique pour maîtriser son budget ", il est indiqué que des CESF du Centre d'action sociale de la Ville de Paris ont participé ; sur un autre, c'est l'association Familles rurales qui a été partenaire, etc.

8. Crédit responsable est désormais la "signature» de Cetelem, qui a déposé le slogan, d'où la présence de la marque ${ }^{\circledR}$ lorsque l'enseigne utilise le terme.

9. Union nationale des centres communaux d'action sociale.

10. Gloukoviezoff et Rebière (2013), dans leur rapport sur l'impact du microcrédit personnel, précisent que les objets financés sont des véhicules, des formations, des dépenses liées au logement, à la santé, ainsi qu'à des besoins professionnels. 
11. Par exemple, le forum annuel du Crédit municipal (octobre 2013) était intitulé « Fragilités sociales : nouveaux publics, nouvelles pratiques ? » et réunis-sait des chercheurs, des représentants bancaires, des représentants de services sociaux et des représen-tants d'associations caritatives comme la Fondation Abbé Pierre.

12. "Avant» étant ici indéterminé, puisque la procédure de surendettement existe depuis 1989 et que cette figure d'une catégorie sociale intermédiaire entre les plus pauvres, aidés, et les plus riches, nan-tis, s'est progressivement constituée depuis plusieurs dizaines d'années. Olivier Schwartz (2009) parle de « catégories modestes » pour les désigner.

13. Les entreprises d'insertion appartiennent au secteur marchand mais ont une vocation sociale et emploient en général pour des durées de six mois à deux ans des personnes très éloignées de l'emploi, qui y sont embauchées et qui, parallèlement à leur travail, y suivent des formations et y bénéficient d'un accompagnement social.

14. Ana Perrin-Heredia (2010) a montré le travail de certaines conseillères en économie sociale et familiale, qui est véritablement un travail de transformation des pratiques budgétaires. Toutefois, la plupart des travailleurs sociaux se contentent d'un rapport très distant au budget des personnes, y compris une partie des CESF dont les postes n'impliquent pas d'accompagnement budgétaire.

15. L'hexis des formateurs de Finances \& Pédagogie est souvent très proche de celle des banquiers d'agence : costume, mallette, coiffure et rasage (pour les hommes) « nets ». 